\title{
Computer-assisted ultrasound quantification analysis in fatty liver model of rats
}

\author{
Zhe Lou, Chunyan Wang, Qiaobei Li, Yinyan Li \\ Department of Ultrasonic Diagnosis, The First Affiliated Hospital of China Medical University, Shenyang, China \\ Contributions: (I) Conception and design: Z Lou, Y Li; (II) Administrative support: Y Li, Q Li; (III) Provision of study materials or patients: Z Lou, \\ C Wang, Q Li; (IV) Collection and assembly of data: Z Lou, C Wang, Q Li; (V) Data analysis and interpretation: C Wang, Q Li; (VI) Manuscript \\ writing: All authors; (VII) Final approval of manuscript: All authors. \\ Correspondence to: Yinyan Li. Department of Ultrasonic Diagnosis, The First Affiliated Hospital of China Medical University, Shenyang, China. \\ Email: liyinyan_dr@163.com.
}

Background Early detection of nonalcoholic fatty liver disease (NAFLD) shows its significant efficacy in
preventing the patients from liver failure. The ultrasonic image quantitative analysis software can assist to
diagnose NAFLD in the clinical studies. In this study, we aim to explore new method to evaluate the value of
computer-assisted ultrasound in diagnosis and classification of fatty liver via Image J software.
Methods: Forty Sprague-Dawley rats were randomly divided into control group (n=10) and model group
(n=30). For model group, the rats received high fat diet and subcutaneous injection of carbon tetrachloride
to establish nonalcoholic fatty liver model. Ultrasound and pathological examinations on rats were
performed on 4,8 , and 12 weeks. Image J software was used to measure the liver grayscale value (LGV)
and renal grayscale value (RGV). The difference between LGV and RGV (LRGV) was analyzed. The
diagnostic performance of computer-assisted ultrasound quantification was evaluated by receiver operating
characteristic (ROC) analysis.

Results: We compared ultrasonic quantization parameters between control and model groups and found that the LGV and LRGV were statistically different between the normal and light fatty livers, light and moderate fatty livers, as well as moderate and severe fatty livers, respectively $(\mathrm{P}<0.05)$. There was no significant difference in RGV among these groups $(\mathrm{P}>0.05)$. Kappa statistic and Bland-Altman analyses showed the consistency of ultrasonic examination and pathological examination was good in diagnosis of fatty liver.

Conclusions: This study indicated that the computer-assisted ultrasound quantification analysis, with high performance of NAFLD diagnosis like pathological examination, could provide a new and flexible noninvasive method for preclinical pharmacological research and basic research.

Keywords: Fatty liver; ultrasound; Image J; liver grayscale value (LGV); renal grayscale value (RGV)

Submitted Nov 26, 2019. Accepted for publication Sep 30, 2020.

doi: 10.21037/apm-19-545

View this article at: http://dx.doi.org/10.21037/apm-19-545

\section{Introduction}

With the change of life style, the incidence rate of nonalcoholic fatty liver diseases (NAFLD) is increasing in recent years, and it has become the most common cause of chronic liver disease in the world, with a global prevalence of $24 \%$ in the general population (1-3). NAFLD arises a major health concern, as it is highly associated with obesity and diabetes and cause considerable liver-related mortality and morbidity $(4,5)$. In fact, if the patients with NAFLD are diagnosed in the early stage, and effective clinical treatments and intervention are carried out in time, usually the liver damage can be reversible (6). Therefore, early detection of NAFLD shows its significant importance in preventing the 
patients from liver cirrhosis and liver failure, and reducing the pain and burden from the patients (5).

At present, liver biopsy and pathological examination are the gold standards for diagnosis of NAFLD $(7,8)$. However, there are still some limitations of these techniques for NAFLD diagnosis. For example, these examination methods are invasive, which will bring some complications that associated with the operative procedure in the patients. Meanwhile, the distribution of fatty liver is quite heterogeneous, and biopsy of liver tissue only accounts for one in one million of the liver tissue. This will bring concerns of tissue sampling variability and increase the false negative rate in patients $(7,9)$. Therefore, additional approaches for noninvasive diagnosis of NAFLD with high efficacy are still desirable in the clinical settings.

Recent accumulated studies have shown that ultrasound, a non-invasively imaging technique with good stability, has become a widely-used method for diagnosis of NAFLD in the clinic $(10,11)$. However, as limited by the specialist experience and instrumentation, the objectivity of diagnosis results is relatively low. In addition, there is no quantitative index for classification of results from NAFLD diagnosis. Although ultrasonic image quantitative analysis software has been employed to diagnose fatty liver in the clinical studies $(12,13)$, the potential application of Image J software (Image J bundled with 64-bit Java 1.8.0_112, Wayne Rasband, National Institute of Mental Health, Bethesda, Maryland, USA) on the computer for analysis of ultrasonic images and obtaining quantitative grading diagnosis of NAFLD is still not fully applied in the clinic. Recent studies have revealed that this method can quantitatively evaluate the degree of fatty liver by comparing the gray scale median of liver and kidney accurately. Another advantage of this method is that it can analyze ultrasonic images offline, which can improve the efficiency and cost-performance value as it allows clinician to assess the ultrasound results with enough time.

In this study, we aim to explore proper quantitative ultrasonic index for classification of diagnosis of fatty liver via Image $\mathrm{J}$ software on the computer, and thus provide new approach to improve the accuracy and stability of ultrasonography for NAFLD diagnosis.

We present the following article in accordance with the ARRIVE reporting checklist (available at http://dx.doi. org/10.21037/apm-19-545).

\section{Methods}

\section{Animal establishment and grouping}

Forty male Sprague-Dawley rats (6-12 weeks, 175 $25 \mathrm{~g})$ were purchased from China Medical University, and maintained in the Experimental Animal Center at China Medical University under specific pathogen-free conditions. All the animal experiments reported herein were carried out in accordance with the approved guideline and approved by the committee on the Animal Care and Use of Institute of China Medical University. Noting that the reason for choosing rat here was because the rat has large mass of liver and kidney and it is more easily examined by ultrasound. Briefly, rats were randomly divided into control group $(\mathrm{n}=10)$ and model group $(\mathrm{n}=30)$ by an investigator who was blinded to this study. Rats in normal control group received basic food daily, while rats in model group received high-fat diet daily as well as subcutaneous injection of carbon tetrachloride solution $(0.05 \mathrm{~mL} / \mathrm{kg}$, China National Pharmaceutical Group Corporation, Shenyang, China) from twice a week for 4 weeks to establish nonalcoholic fatty liver model (14-16).

\section{Ultrasonic examination and parameter analysis}

Before the ultrasonic examination, rats were fasted overnight. The chest and abdomen were depilated with $8 \% \mathrm{Na}_{2} \mathrm{~S}$ solution (China National Pharmaceutical Group Corporation, Shenyang, China). After that, rats were anesthetized by an intraperitoneal injection of $4 \%$ chloral hydrate (China National Pharmaceutical Group Corporation, Shenyang, China), and then placed in the supine position. Ultrasound diagnostic instrument and probe (L12-5) (PHILIPS iU22, The Netherlands) were applied to examine the rat liver and kidney conditions in this study. Under MSK Sup, a total gain was 88 dB/DR50, the depth was $2.5 \mathrm{~cm}$, resolution and sensitivity were fixed, and the time gain compensate (TGC) curve adjustment knob was placed in a straight line for ultrasonic examination. The rat liver was observed in the intercostal transverse longitudinally. Ultrasonic images of rat liver and kidney were taken and compared. After that, the images were imported to the computer through image conduction function of PHILIPS iU22, and opened through Image J software. 


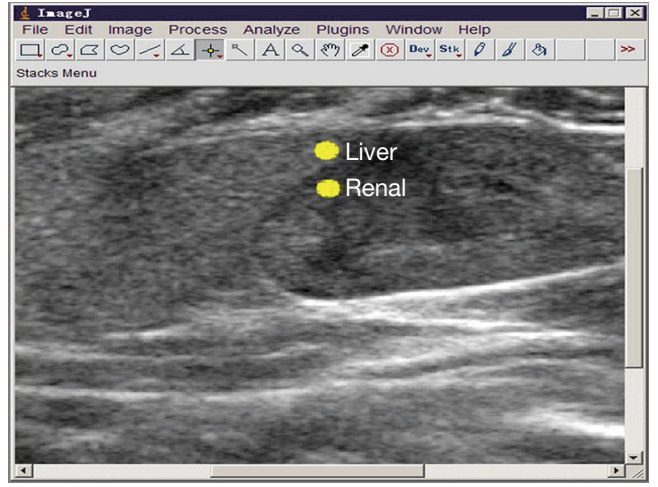

Figure 1 ROI placement on rat liver and kidney in an ultrasonic image by Image J software. One ROI was placed in the liver parenchyma. The other ROI was placed on the junction of the renal cortex medulla. The two ROIs were kept on the same vertical line. ROI, region of interest.

Table 1 Comparison of pathological diagnosis result and ultrasonic examination using LGV parameter in rats

\begin{tabular}{lcccc}
\hline Degree & $\begin{array}{c}\text { Pathological } \\
\text { diagnosis }(\mathrm{n})\end{array}$ & $\begin{array}{c}\text { Ultrasound } \\
\text { diagnosis }(\mathrm{n})\end{array}$ & P value & $\begin{array}{c}\text { kappa } \\
\text { value }\end{array}$ \\
\hline NFL & 11 & 8 & $>0.05$ & 0.747 \\
LFL & 10 & 13 & $>0.05$ & 0.570 \\
MFL & 10 & 7 & $>0.05$ & 0.748 \\
SFL & 9 & 12 & $>0.05$ & 0.881 \\
\hline
\end{tabular}

LGV, gray liver value; NFL, no fatty liver; LFL, light fatty liver; MFL, moderate fatty liver; SFL, severe fatty liver.

Two circular region of interests (ROIs) with $330 \mathrm{~mm}^{2}$ were applied on liver and kidney, respectively (Figure 1). One ROI was placed in the liver parenchyma $(1.0 \pm 0.5 \mathrm{~cm}$ from skin). The other ROI was placed on the junction of the renal cortex medulla ( $50 \%$ cortex and $50 \%$ medulla), which was away from renal column. The two ROIs were kept on the same vertical line. Liver grayscale value (LGV) and renal grayscale value (RGV) were then measured using Image $\mathrm{J}$ software by two independent researchers, and the average value was collected for further analysis. The difference between LGV and RGV (LRGV) was calculated.

\section{Pathological examination}

The right lobe of livers $(1 \mathrm{~cm} \times 1 \mathrm{~cm} \times 0.5 \mathrm{~cm})$ were harvested and fixed in $4 \%$ Paraformaldehyde solution (China National Pharmaceutical Group Corporation,
Shenyang, China). After paraffin-embedding and sectioning of tissues, the obtained slices ( $5 \mu \mathrm{m}$ thickness) were stained with hematoxylin-eosin (H\&E) staining. After that, five random slices were selected, and mounted with an aqueous mounting medium. The pathological grades were determined according to the proportion of fat infiltrated hepatocytes in the total number of hepatocytes (17): less than $30 \%$ was considered as light fatty liver disease (FLD); $30-60 \%$ was considered as moderate FLD; and more than $60 \%$ was considered as severe FLD.

\section{Statistical analysis}

The data were statistically analyzed using SPSS statistical analysis software (version 13.0, SPSS Inc, Chicago, IL, USA). Data were presented as mean $\pm \mathrm{SD}$. One way analysis of variance (ANOVA) was used to compare the differences among different groups, and a $\mathrm{P}$ value $<0.05$ was considered statistically significant. Intra-observer agreement was evaluated using kappa statistic, and values in the ranges of $0.01-0.20,0.21-0.40,0.41-0.60,0.61-0.80$, and $0.81-1.00$ were considered as slight, fair, moderate, substantial, and almost perfect agreement, respectively. The BlandAltman analysis was applied to compare the ultrasonic examination and pathological examination for diagnosis of fat liver. Meanwhile, the maximum points of sensitivity and specificity were selected as the diagnostic thresholds according to the receiver operating characteristic (ROC) curve. Area under the curve (AUC) was used to evaluate the diagnostic performance of computer-assisted ultrasound quantification analysis in different groups.

\section{Results}

\section{Ultrasound results}

The normal, light fatty, moderate fatty, and severe fatty liver in rats were evaluated by ultrasonic examination and Image J software. Two ROIs were placed on liver and kidney on ultrasonic images, respectively, as to obtain the values of LGV and RGV. The LGV, RGV, and the difference between LGV and RGV (LRGV) were analyzed. When using LGV parameter to determine the grades of fatty liver, it showed that 8 rats with no fatty liver, 13 rats with light fatty liver, 7 rats with moderate fatty liver and 12 rats with severe fatty livers (Table 1). When using LRGV to determine the grades of fatty liver, it showed that 9 rats with no fatty liver, 14 rats with light fatty liver, 7 rats with 
Table 2 Comparison of pathological diagnosis result and ultrasonic examination using LRGV in rats

\begin{tabular}{lcccc}
\hline Degree & $\begin{array}{c}\text { Pathological } \\
\text { diagnosis }(n)\end{array}$ & $\begin{array}{c}\text { Ultrasound } \\
\text { diagnosis }(n)\end{array}$ & P value & $\begin{array}{c}\text { kappa } \\
\text { value }\end{array}$ \\
\hline NFL & 11 & 9 & $>0.05$ & 0.756 \\
LFL & 10 & 14 & $>0.05$ & 0.676 \\
MFL & 10 & 7 & $>0.05$ & 0.768 \\
SFL & 9 & 10 & $>0.05$ & 0.931 \\
\hline
\end{tabular}

LRGV, the difference between liver gray value and kidney gray value; NFL, no fatty liver; LFL, light fatty liver; MFL, moderate fatty liver; SFL, severe fatty liver.

moderate fatty liver and 10 rats with severe fatty livers (Table 2). The representative ultrasonic images for different grades of fatty liver were shown in Figure 2.

\section{Comparison of ultrasonic quantization parameters among different groups}

Next, the LGV, RGV and LRGV in different groups were statistically analyzed. As shown in Figure 3, the LGV was statistically different between normal and light fatty livers, light and moderate fatty livers, and moderate and severe fatty livers $(\mathrm{P}<0.05)$ (Figure $3 A)$. There was no significant difference in RGV among these groups $(\mathrm{P}>0.05)$ (Figure 3B). The LRGV was statistically different between normal and light fatty livers, light and moderate fatty livers, and moderate and severe fatty livers $(\mathrm{P}<0.05)($ Table 3$)$.

\section{Pathological results}

The pathological grades of FLD in each rat were examined by H\&E staining. The results demonstrated that no fatty liver was observed in control group $(n=10)$. While in model group, there were 1 rat with normal liver, 10 rats with light fatty liver, 10 rats with moderate fatty liver and 9 rats with severe fatty livers. The representative $H \& E$ images of normal, light fatty, moderate fatty, and severe fatty livers were presented in Figure 4, and the fatty cells in the liver were pointed out by yellow arrows. Meanwhile, at the end of the experiment (12 weeks later), the bodyweight of rats in each group was collected and shown in Table 4. The average bodyweight of rats in control group $(241 \pm 22 \mathrm{~g})$ was significantly lower than that in light fatty $(302 \pm 29 \mathrm{~g})$, moderate fatty $(321 \pm 31 \mathrm{~g})$, and severe fatty liver $(354 \pm 29 \mathrm{~g})$ groups $(\mathrm{P}<0.05)$, demonstrating that the bodyweight was associated with the grades of fatty liver in rats.

\section{Consistency of ultrasonic examination and pathological examination}

The comparison of ultrasonic examination and pathological examination was conducted by kappa statistic and Bland-Altman analyses. The kappa values for these two examination in detection of normal, light fatty, moderate fatty, and severe fatty liver of rats were 0.747, 0.570, 0.748, 0.881 by using LGV parameter, respectively (Table 1 ), and $0.756,0.676,0.768$, and 0.931 by using LRGV index, respectively (Table 2). The Bland-Altman analysis results were shown in Figure 5. The difference of pathological examination and ultrasonic examination did not exceed the maximum allowed difference (mean $\pm 1.96 \mathrm{SD}$ ), implying the two examinations were with high consistency for diagnosis of fatty liver. The diagnostic performance of computerassisted ultrasound quantification analysis was evaluated. When using LGV index for assessment, the sensitivity and specificity were $75 \%$ and $87.5 \%$ for the diagnosis of normal liver and light fatty liver respectively; and $77.8 \%$ and $85.8 \%$ for the diagnosis of light fatty liver and moderate fatty liver respectively; and $77.8 \%$ and $88.9 \%$ for moderate fatty liver and severe fatty liver respectively (Table 5). When using LRGV index for assessment, the sensitivity and specificity were $91.7 \%$ and $91.7 \%$ for diagnosis of normal liver and light fatty liver respectively; and $90.0 \%$ and $90.2 \%$ for light fatty liver and moderate fatty liver respectively; and $87.1 \%$ and $90.3 \%$ for moderate fatty liver and severe fatty liver respectively (Table 6).

\section{The diagnostic threshold of quantitative parameter}

Based on ROC statistical analysis, the accuracy of the diagnostic test was evaluated by the area under the ROC curve (AUC). The sensitivity and specificity of ultrasound examination by using LGV and LRGV as index to compare the difference between different groups were calculated and plotted on the ROC curves (Figure 6). The AUC value of LGV in light FLD, moderate FLD, and severe FLD was $0.875,0.808,0.860$, respectively (Table 5), and the LRGV in light FLD, moderate FLD, and severe FLD was 0.885 , 0.909, 0.950, respectively (Table 6), which demonstrated high diagnostic performance of computer-assisted ultrasound quantification analysis in these groups. 

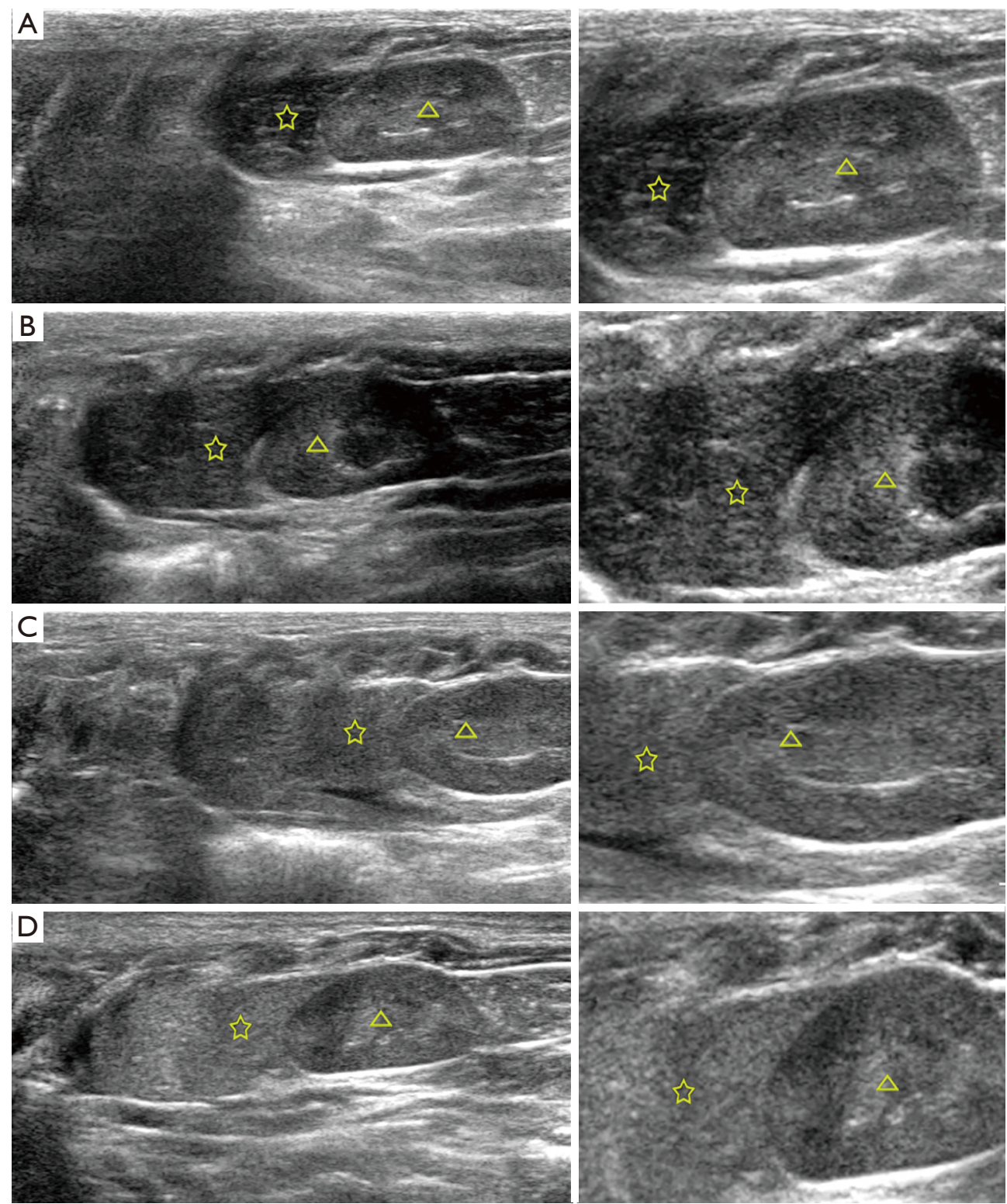

Figure 2 The morphology of different pathological grades of fatty liver determined by ultrasound examination. (A) The normal liver; (B) the light fatty liver; (C) the moderate fatty liver; (D) the severe fatty liver. Yellow stars represented liver tissue and yellow triangles represented kidney tissue.

\section{Discussion}

As a noninvasive and reliable diagnosis technique, ultrasound has become a preferred method for diagnosis of fatty liver in the clinical practice, and with an overall sensitivity of $85 \%$ and specificity of $94 \%(11,18,19)$. Besides ultrasonography, there are some other noninvasive approaches, such as computed tomography (CT), magnetic resonance (MR), magnetic resonance elastography
(MRE), that have been employed to detect fatty liver and grades (18). CT-based diagnosis of fatty liver is considered as accurate, however, as it requires radiation and is outperformed by dual-gradient echo MRI, CT is not recommended for the diagnosis of fatty liver (20). MR imaging is more sensitive than CT for hepatic steatosis diagnosis, and generally considered the effective radiologic modality for qualitative and quantitative assessment of fatty 

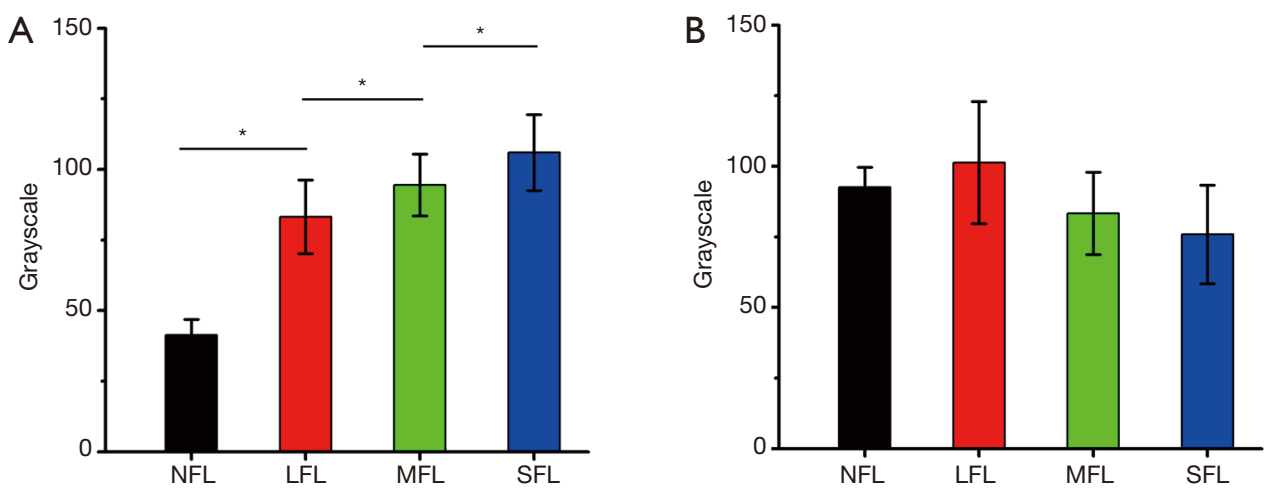

Figure 3 The values of LGV and RGV in different grades of fatty liver groups. The gray values of ROCs placed in liver and kidney were determined by Image J software. (A) LGV; (B) RGV. Data were presented as mean \pm SD. *, P<0.05. LGV, liver grayscale value; RGV, renal grayscale value.

Table 3 Quantitative index of fatty liver in rats (mean \pm SD)

\begin{tabular}{|c|c|c|c|c|}
\hline Degree & $\mathrm{N}$ & LGV & RGV & LRGV \\
\hline NFL & 11 & $41.40 \pm 5.50$ & $92.62 \pm 7.00$ & $-49.22 \pm 10.71$ \\
\hline LFL & 10 & $83.22 \pm 13.07^{\mathrm{a}}$ & $101.32 \pm 21.59^{d}$ & $-18.10 \pm 15.94^{a}$ \\
\hline MFL & 10 & $94.49 \pm 10.89^{b}$ & $83.32 \pm 14.62^{d}$ & $11.17 \pm 9.02^{b}$ \\
\hline SFL & 9 & $105.96 \pm 13.48^{c}$ & $75.87 \pm 17.48^{\mathrm{e}}$ & $33.12 \pm 8.17^{c}$ \\
\hline \multicolumn{5}{|c|}{$\begin{array}{l}{ }^{a} \text {, LFL vs. NFL, } P<0.05 ;{ }^{b}, M F L \text { vs. LFL, } P<0.05 ;{ }^{c}, S F L \text { vs. MFL, } \\
P<0.05 ;{ }^{d} \text {, MFL vs. LFL, P }>0.05 ;{ }^{e}, S F L \text { vs. MFL, } P>0.05 . L G V \text {, } \\
\text { liver gray value; RGV, renal gray value; LRGV, the difference } \\
\text { between liver and kidney; NFL, no fatty liver; LFL, light fatty } \\
\text { liver; MFL, moderate fatty liver; SFL, severe fatty liver. }\end{array}$} \\
\hline
\end{tabular}

liver but is relatively costly (21). MRE is more accurate for the detection of early NAFLD and its stages, with a cutoff of 3.64 for advanced liver fibrosis; while it is also the most expensive technique for non-invasive detection of fatty liver and requires special external equipment and software (22). What's more, blood biochemical biomarkers have been extensively studied for NAFLD diagnosis, including fibrosis-4 (FIB-4) and NAFLD fibrosis score (NFS); however, the sensitivity and specificity of these tests are lower for earlier and more moderate stages of fatty liver $(18,23)$. Compared with these noninvasive methods, ultrasound has high cost performance. Unlike CT and MR, liver iron has little effect on the ultrasound beam (24). However, Operator and machine dependency are the major drawbacks of ultrasonography. The sensitivity and specificity of ultrasonography are more accurate at detecting moderate to severe fatty liver, but will drop to $65 \%$ and $81 \%$ when all grads are considered $(19,24)$.
The liver is a vital organ of the lipid metabolism. Fat metabolism disorder has a great influence for dynamic balance of lipids. When the fat storage volume exceeds more than $5 \%$ of the liver mass, fat will be stored in hepatocytes. If more than $33 \%$ fatty degeneration, it is called fatty liver. According to hepatocyte fat storage volume, the grades of fatty liver can be classified as light, mild and severe ones (25). Ultrasonic characteristics for fatty liver are due to hepatic fat deposition in the cells, obvious ultrasonic energy absorption and scattering effect. The sound energy is mainly absorbed by the former site of liver, and obviously attenuated in the back site of liver, which formed the socalled bright liver (26). However, the attenuation of hepatic ultrasound images was not obviously observed in this study. This may because the probe L12-5 had a strong ability of penetration into the liver. In addition, a mild fibrosis also can reduce the degree of attenuation.

Currently, a computer-aided diagnostic system was used to objectively measure and classify the different grades of FLD, such as gray-level co-occurrence matrix (27), support vector machine (28), probabilistic neural network (29), extreme learning machine framework (30). In our study, we combined ultrasound and Image J software to diagnose and classify the grades of the fatty liver in rats. Single index, such as LGV and LRGV of ultrasound images, were used to assess the classification of FLD. We compared the rat model of fatty liver through computer-assisted ultrasound quantification and pathological diagnosis, and confirmed the feasibility and stability of quantification ultrasonography analysis in FLD diagnosis. More importantly, the Image J software on the computer showed its great ability to analyze ultrasonic images for fatty liver diagnosis and classification. 

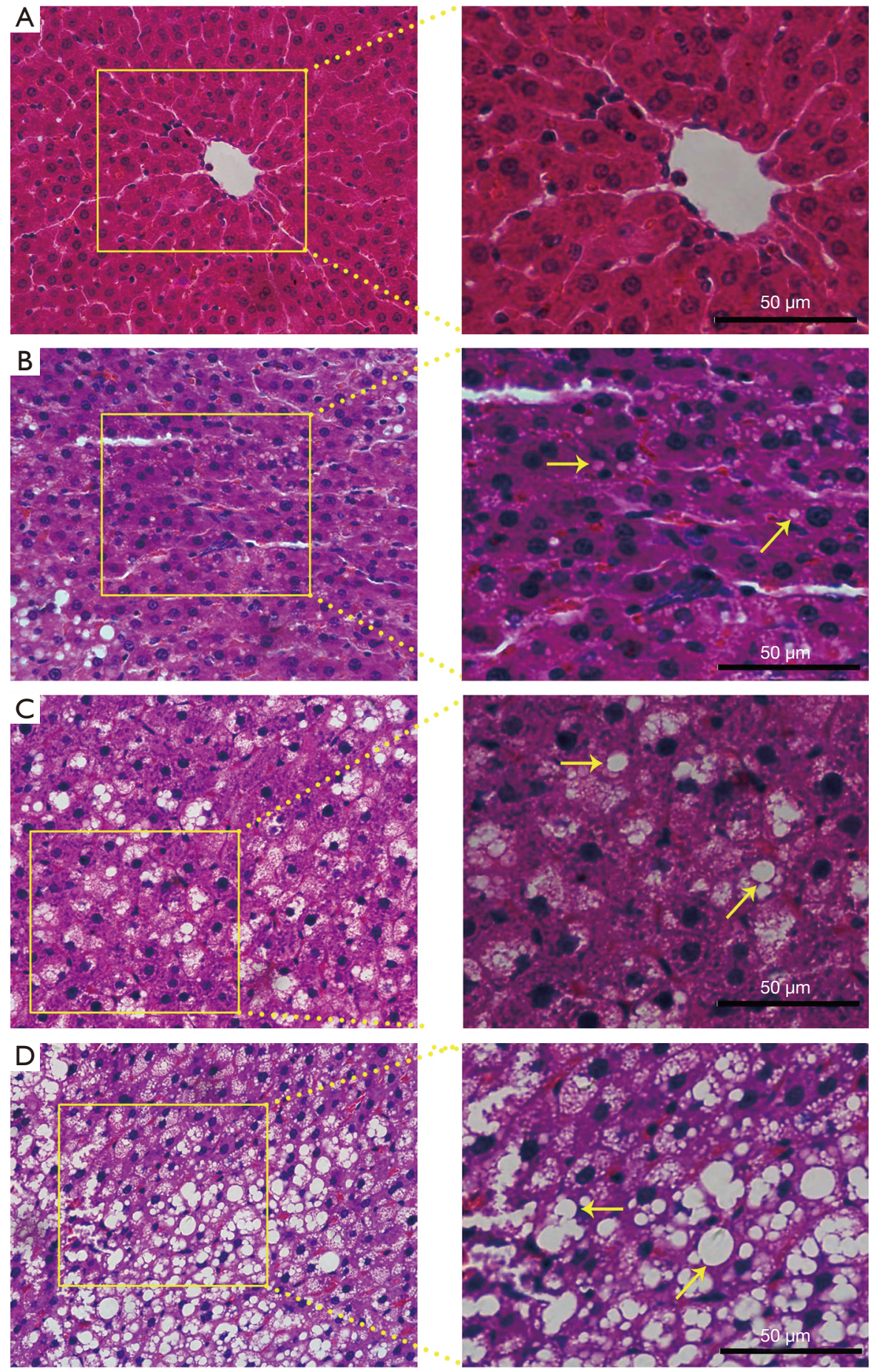

Figure 4 The pathological change of different grades of fatty liver in rats. H\&E staining was used to stain the liver tissues, and the pathological grades were determined according to the proportion of fat infiltrated hepatocytes. The representative images were taken at 400x magnification. (A) The normal liver; (B) the light fatty liver; (C) the moderate fatty liver; (D) the severe fatty liver. The fatty cells in the liver were pointed out by yellow arrows. 
Table 4 The bodyweight of rats at the end of the experiment

\begin{tabular}{lcccc}
\hline Degree & $\mathrm{n}$ & Minimum $(\mathrm{g})$ & Maximum $(\mathrm{g})$ & Mean \pm SD $(\mathrm{g})$ \\
\hline NFL & 11 & 204 & 276 & $241 \pm 22$ \\
LFL & 10 & 261 & 349 & $302 \pm 29^{\mathrm{a}}$ \\
MFL & 10 & 285 & 375 & $321 \pm 31^{\mathrm{b}}$ \\
SFL & 9 & 294 & 400 & $354 \pm 29^{\mathrm{c}, \mathrm{d}, \mathrm{e}}$ \\
\hline
\end{tabular}

${ }^{a}$, LFL vs. NFL, $\mathrm{P}<0.05 ;{ }^{b}, \mathrm{MFL}$ vs. NFL, $\mathrm{P}<0.05 ;{ }^{\mathrm{c}}, \mathrm{SFL}$ vs. NFL, $\mathrm{P}<0.05 ;{ }^{\mathrm{d}}, \mathrm{SFL}$ vs. LFL, $\mathrm{P}<0.05 ;{ }^{\mathrm{e}}, \mathrm{SFL}$ vs. MFL, $\mathrm{P}<0.05$. NFL, no fatty liver; LFL, light fatty liver; MFL, moderate fatty liver; SFL, severe fatty liver.
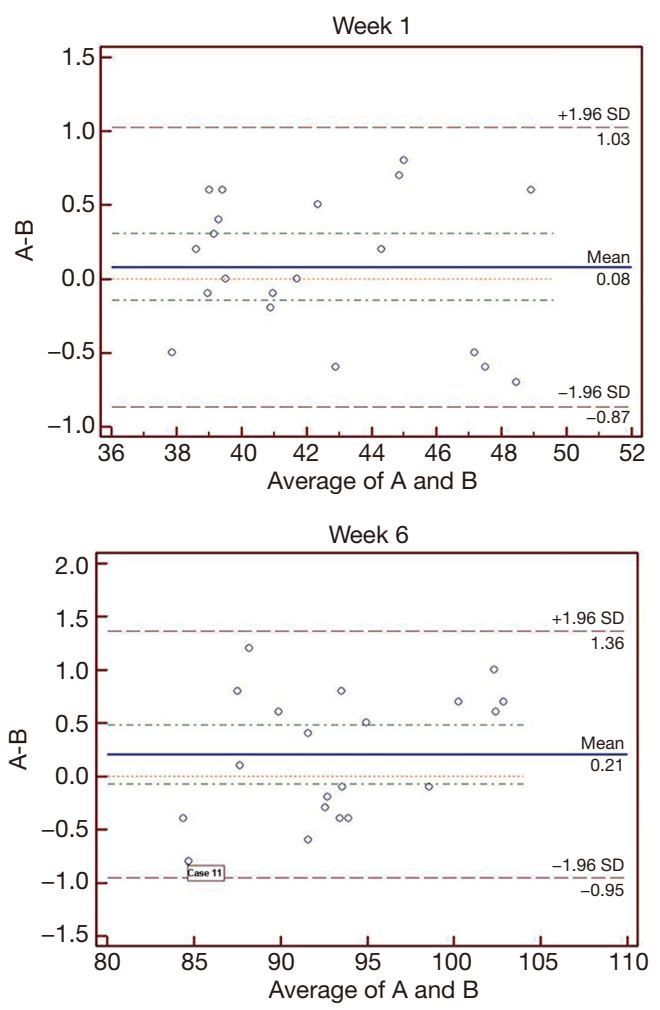

Figure 5 Comparison of ultrasonic examination and pathological examination by Bland-Altman analysis. A represented pathological examination and $\mathrm{B}$ represented ultrasonic examination.

This combined approach demonstrated a simpler and easier way with high sensitivity and specificity of performance when compared with the above methods which ultrasound images are evaluated via software-embedded in ultrasonic machine (31).

Previous studies showed that the diagnostic sensitivity of ultrasonic examination for moderate fatty liver was more
Table 5 The diagnostic performance of LGV

\begin{tabular}{lcccc}
\hline Degree & AUC & Threshold & Sensitivity (\%) & Specificity (\%) \\
\hline LFLD & 0.875 & 52.9 & 75.0 & 87.5 \\
MFLD & 0.808 & 94.9 & 77.8 & 85.8 \\
SFLD & 0.860 & 101.1 & 77.8 & 88.9 \\
\hline
\end{tabular}

LGV, liver gray value; AUC, area under the curve; LFLD, light fatty liver disease; MFLD, moderate fatty liver disease; SFLD, severe fatty liver disease.

Table 6 The diagnostic performance of LRGV

\begin{tabular}{lcccc}
\hline Degree & AUC & Threshold & Sensitivity (\%) & Specificity (\%) \\
\hline LFLD & 0.885 & -28.4 & 91.7 & 91.7 \\
MFLD & 0.909 & 10.9 & 90.0 & 90.2 \\
SFLD & 0.950 & 24.5 & 87.1 & 90.3 \\
\hline
\end{tabular}

LRGV, the quantitative joint index (liver to kidney); AUC, area under the curve; LFLD, light fatty liver disease; MFLD, moderate fatty liver disease; SFLD, severe fatty liver disease.

than $90 \%$, but only $30-64 \%$ for light fatty liver $(19,24,32)$. In this study, we demonstrated that the sensitivity and specificity of ultrasonic examination by Image $J$ software analysis of LGV and RGV were both very high. In addition, the quantitative joint index (LRGV) was proved to effectively act as a classification index for the FLD diagnosis, and offline image analysis software was allowed for ultrasonic results analysis. The obtained technique could provide a high cost performance and stability method for FLD classification, and exhibit their advantages over other related techniques.

There were still several limitations in this study. Firstly, only a small number of samples were analyzed, and further investigations with large number are required to reinforce this study. It has been reported that the degree of liver fibrosis can affect the sensitivity of the diagnosis, and therefore application of related parameter can reduce the influence of individual differences, but it is not enough for the filter degree under different environment. Although the parameters of the depth, gain, time compensation and gain compensation for each ultrasonic operation are fixed, the measuring sites, focal length settings and scanning sections for animal examination have inevitable influences for the results. When ROI in ultrasonic image was selected by using Image $\mathrm{J}$ software, the ROI of RGV was placed directly below that of LGV. This would result in incorrect 
A

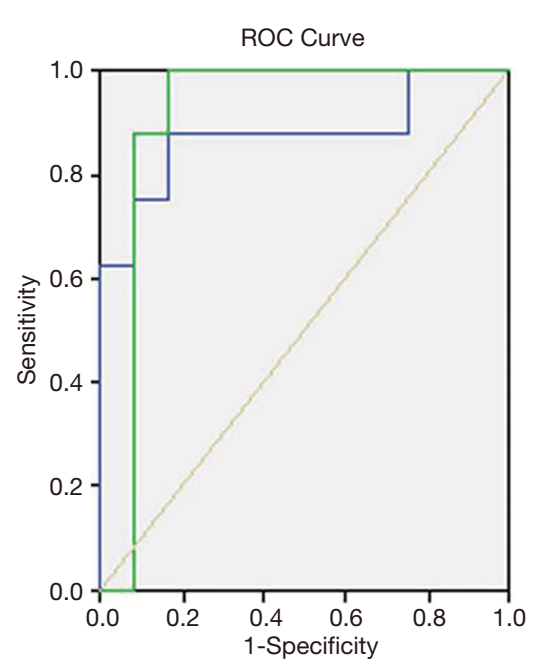

B

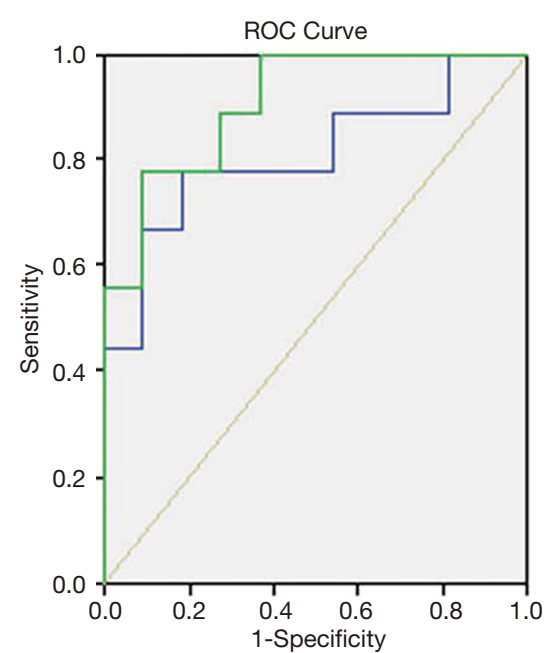

C

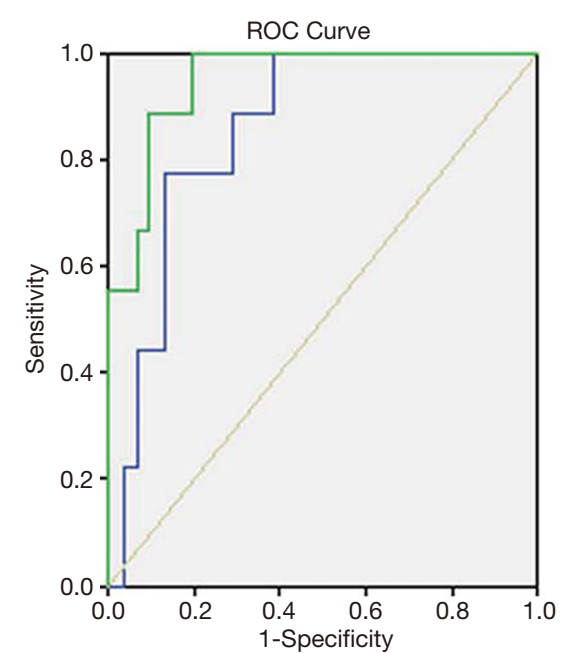

Figure 6 Evaluation of the accuracy of the diagnostic test by ROC curve. (A) The ROC curve of LGV and LRGV between NAFLD group and LFLD group; (B) the ROC curve of LGV and LRGV between LFLD group and MFLD group; (C) the ROC curve of LGV and LRGV between MFLD group and SFLD group. ROC, receiver operating characteristic; LGV, liver grayscale value; NAFLD, nonalcoholic fatty liver disease; LFLD, light fatty liver disease; MFLD, moderate fatty liver disease; SFLD, severe fatty liver disease.

calculation of LRGV as LGV and RGV came from the same sound beam. Therefore, the LRGV would be greatly influenced by different ultrasonic instrument, different section, abdominal gas and pseudomorphism.

\section{Conclusions}

In conclusion, our study indicated that both LGV and LRGV can be used as the index for diagnosis of fatty liver in rats. LRGV can also be used to as the index for classification diagnosis. Computer-Assisted Ultrasound Quantification Analysis may provide a new and flexible noninvasive method for preclinical pharmacological research and basic research.

\section{Acknowledgments}

Funding: None.

\section{Footnote}

Reporting Checklist: The authors have completed the ARRIVE reporting checklist. Available at http://dx.doi. org/10.21037/apm-19-545
Data Sharing Statement: Available at http://dx.doi. org/10.21037/apm-19-545

Conflicts of Interest: All authors have completed the ICMJE uniform disclosure form (available at http://dx.doi. org/10.21037/apm-19-545). The authors have no conflicts of interest to declare.

Ethical Statement: The authors are accountable for all aspects of the work in ensuring that questions related to the accuracy or integrity of any part of the work are appropriately investigated and resolved. All the animal experiments reported herein were carried out in accordance with the approved guideline and approved by the committee on the Animal Care and Use of Institute of China Medical University.

Open Access Statement: This is an Open Access article distributed in accordance with the Creative Commons Attribution-NonCommercial-NoDerivs 4.0 International License (CC BY-NC-ND 4.0), which permits the noncommercial replication and distribution of the article with the strict proviso that no changes or edits are made and the original work is properly cited (including links to both the 
formal publication through the relevant DOI and the license). See: https://creativecommons.org/licenses/by-nc-nd/4.0/.

\section{References}

1. Rosso N, Bellentani S. Nonalcoholic Fatty Liver Disease: A Wide Spectrum Disease. Liver Disease 2020;273-84.

2. Younossi Z, Tacke F, Arrese M, et al. Global perspectives on nonalcoholic fatty liver disease and nonalcoholic steatohepatitis. Hepatology 2019;69:2672-82.

3. Pappachan JM, Babu S, Krishnan B, et al. Non-alcoholic Fatty Liver Disease: A Clinical Update. J Clin Transl Hepatol 2017;5:384-93.

4. Targher G, Byrne CD, Lonardo A, et al. Non-alcoholic fatty liver disease and risk of incident cardiovascular disease: A meta-analysis. J Hepatol 2016;65:589-600.

5. Simeone JC, Bae JP, Hoogwerf BJ, et al. Clinical course of nonalcoholic fatty liver disease: an assessment of severity, progression, and outcomes. Clin Epidemiol 2017;9:679-88.

6. Bao Y. The progress of studying the mechanisms of immune cells in the regulation of non-alcoholic fatty liver diseases. Zhonghua Gan Zang Bing Za Zhi 2017;25:553-6.

7. Brunt EM. Nonalcoholic fatty liver disease and the ongoing role of liver biopsy evaluation. Hepatol Commun 2017;1:370-8.

8. Review Team, LaBrecque DR, Abbas Z, et al. World Gastroenterology Organisation. World Gastroenterology Organisation global guidelines: Nonalcoholic fatty liver disease and nonalcoholic steatohepatitis. J Clin Gastroenterol 2014;48:467-73.

9. Nalbantoglu IL, Brunt EM. Role of liver biopsy in nonalcoholic fatty liver disease. World J Gastroenterol 2014;20:9026-37.

10. Dandan L, Huanhuan M, Xiang L, et al. Classification of diffuse liver diseases based on ultrasound images with multimodal features. 2019 IEEE International Instrumentation and Measurement Technology Conference (I2MTC) 2019;1-5.

11. Morikawa H, Mano K, Horinaka H, et al. Development of non-invasive method for assessment of hepatic steatosis. Ultrasonics 2016;72:195-200.

12. Benjamin A, Zubajlo R, Thomenius K, et al. Non-invasive diagnosis of non-alcoholic fatty liver disease (NAFLD) using ultrasound image echogenicity. Annu Int Conf IEEE Eng Med Biol Soc 2017;2017:2920-3.

13. Kumagai H, Yokoyama K, Katsuyama K, et al. A new method for measuring the speed of sound in rat liver ex vivo using an ultrasound system: correlation of sound speed with fat deposition. Ultrasound Med Biol 2014;40:2499-507.

14. Zheng YP, Zhong XY, Huang YS, et al. HCBP6 Is Involved in the Development of Hepatic Steatosis Induced by High-Fat Diet and CCL4 in Rats. Ann Hepatol 2018;17:511-8.

15. Van Herck MA, Vonghia L, Francque SM. Animal Models of Nonalcoholic Fatty Liver Disease-A Starter's Guide. Nutrients 2017;9:1072.

16. Wahid A, Hamed AN, Eltahir HM, et al. Hepatoprotective activity of ethanolic extract of Salix subserrata against CCl4-induced chronic hepatotoxicity in rats. BMC Complement Altern Med 2016;16:263.

17. Liu F, Xiang Z, Xu J, et al. Establishment of a nonalcoholic fatty liver mice model with high-fat feeding and carbon tetrachloride. Jiangsu Medical Journal 2010;36:69-71.

18. Stefan N, Häring HU, Cusi K. Non-alcoholic fatty liver disease: causes, diagnosis, cardiometabolic consequences, and treatment strategies. Lancet Diabetes Endocrinol 2019;7:313-24.

19. Moreno C, Mueller S, Szabo G. Non-invasive diagnosis and biomarkers in alcohol-related liver disease. J Hepatol 2019;70:273-83.

20. Lee SS, Park SH, Kim HJ, et al. Non-invasive assessment of hepatic steatosis: prospective comparison of the accuracy of imaging examinations. J Hepatol 2010;52:579-85.

21. Abd El-Kader SM, EI-Den Ashmawy EMS. Non-alcoholic fatty liver disease: The diagnosis and management. World J Hepatol 2015;7:846-58.

22. Xiao G, Zhu S, Xiao X, et al. Comparison of laboratory tests, ultrasound, or magnetic resonance elastography to detect fibrosis in patients with nonalcoholic fatty liver disease: A meta-analysis. Hepatology 2017;66:1486-501.

23. Loomba R. Role of imaging-based biomarkers in NAFLD: Recent advances in clinical application and future research directions. J Hepatol 2018;68:296-304.

24. Zhang YN, Fowler KJ, Hamilton G, et al. Liver fat imaging-a clinical overview of ultrasound, CT, and MR imaging. Br J Radiol 2018;91:20170959.

25. Takahashi Y, Fukusato T. Histopathology of nonalcoholic fatty liver disease/nonalcoholic steatohepatitis. World J Gastroenterol 2014;20:15539-48.

26. Liao YY, Yang KC, Lee MJ, et al. Multifeature analysis of an ultrasound quantitative diagnostic index for classifying nonalcoholic fatty liver disease Sci Rep 2016;6:35083.

27. Owjimehr M, Danyali H, Helfroush MS, et al. Staging of 
Fatty Liver Diseases Based on Hierarchical Classification and Feature Fusion for Back-Scan-Converted Ultrasound Images. Ultrason Imaging 2017;39:79-95.

28. Subramanya MB, Virmani J. A DEFS Based System for Differential Diagnosis Between Severe Fatty Liver and Cirrhotic Liver Using Ultrasound Images. Machine Learning in Bio-Signal Analysis and Digaonistic Imaging 2019:53-72.

29. Acharya UR, Raghavendra U, Fujita H, et al. Automated characterization of fatty liver disease and cirrhosis using curvelet transform and entropy features extracted from ultrasound images. Comput Biol Med 2016;79:250-8.

Cite this article as: Lou Z, Wang C, Li Q, Li Y. Computerassisted ultrasound quantification analysis in fatty liver model of rats. Ann Palliat Med 2021;10(2):1631-1641. doi: 10.21037/apm-19545
30. Kuppili V, Biswas M, Sreekumar A, et al. Extreme Learning Machine Framework for Risk Stratification of Fatty Liver Disease Using Ultrasound Tissue Characterization. J Med Syst 2017;42:18.

31. Soder RB, Baldisserotto M, Duval da Silva V. Computerassisted ultrasound analysis of liver echogenicity in obese and normal-weight children. AJR Am J Roentgenol 2009;192:W201-5.

32. Palmentieri B, de Sio I, La Mura V, et al. The role of bright liver echo pattern on ultrasound B-mode examination in the diagnosis of liver steatosis. Dig Liver Dis 2006;38:485-9. 